\title{
INFINITELY MANY SOLUTIONS \\ TO QUASILINEAR ELLIPTIC EQUATION \\ WITH CONCAVE AND CONVEX TERMS
}

\author{
Leran Xia - Minbo Yang - Fukun ZhaO
}

ABSTRACT. In this paper, we are concerned with the following quasilinear elliptic equation with concave and convex terms

(P) $\quad-\Delta u-\frac{1}{2} u \Delta\left(|u|^{2}\right)=\alpha|u|^{p-2} u+\beta|u|^{q-2} u, \quad x \in \Omega$,

where $\Omega \subset \mathbb{R}^{N}$ is a bounded smooth domain, $1<p<2,4<q \leq 22^{*}$. The existence of infinitely many solutions is obtained by the perturbation methods

\section{Introduction}

In the present paper, we are concerned with the following quasilinear elliptic equation with concave and convex terms

$(\mathrm{P})$

$$
\begin{cases}-\Delta u-\frac{1}{2} u \Delta\left(|u|^{2}\right)=\alpha|u|^{p-2} u+\beta|u|^{q-2} u, & x \in \Omega \\ u=0, & x \in \partial \Omega\end{cases}
$$

2010 Mathematics Subject Classification. 35B05, 35B45.

Key words and phrases. Concave and convex terms, fountain theorem, perturbation methods.

The first author was supported by the key programm of Yunnan Normal University, China.

The second author was supported by NSFC (11101374 and 11271331).

The corresponding author was supported partly by NSFC(11061040 and 11361078), Key Project of Chinese Ministry of Education(No212162), NSFY of Yunnan Province (2011CI020) and China Scholarship Council, China. 
where $\Omega \subset \mathbb{R}^{N}$ is a bounded smooth domain, $\alpha, \beta \in \mathbb{R}$ are parameters, $1<p<2$, $4<q \leq 22^{*}, 2^{*}=2 N /(N-2)$ if $N \geq 3$ and $2^{*}=\infty$ if $N=1,2$. Such a problem is referred to as the so-called modified Schrödinger equation (see [3], [24] and [19]). Our motivation comes from the works about the semilinear case (see, for example, [2] and [6])

$$
\begin{cases}-\Delta u=\alpha|u|^{p-2} u+\beta|u|^{q-2} u, & x \in \Omega, \\ u=0, & x \in \partial \Omega,\end{cases}
$$

where $1<p<2<q<2^{*}$. In [2] Ambrosetti, Brezis and Cerami showed that, for $\alpha>0$ small and $\beta>0,(1.1)$ has infinitely many solutions with negative energy and infinitely many solutions with positive energy in $H_{0}^{1}(\Omega)$. In [6] Bartsch and Willem dropped the restriction on $\alpha$ via the fountain theorem and its dual version. More precisely, they show that for $\beta>0$ and $\alpha \in \mathbb{R}$ (1.1) has infinitely many solutions with energy going to infinity, and for $\alpha>0$ and $\beta \in \mathbb{R}$ (1.1) has infinitely many solutions with negative energy going to zero.

In the case $q=2^{*}$, the existence of a positive solution was obtained by Brezis and Nirenberg in [9]. See [17] for the multiplicity result. A natural problem is whether the same conclusions hold true or not for the quasilinear problem $(\mathrm{P})$ ?

In this paper, we will give positive answer for the subcritical case $q<22^{*}$ and the critical case $q=22^{*}$. The main idea of our arguments comes from the works concerning the fountain theorem and its dual version (see [4], [5] and [6]).

Problems similar to $(\mathrm{P})$ were considered recently in some papers. The minimization methods was used in [19], [24]. The main tool in [3], [20] is the Nehari method. A change of variables argument was involved in [11], [21]. With this change of variables the quasilinear problem is transformed to a semilinear problem and various existing methods for semilinear problems can be adopted and modified to treat the resulting equation such as done recently in [1], [11]-[14], [18], [23], [25] and the references therein. In particular, in [13], the authors obtained the existence of a positive solution of a similar problem on $\mathbb{R}^{N}$.

The weak form of $(\mathrm{P})$ is

$$
\int_{\Omega}\left[\left(1+u^{2}\right) \nabla u \nabla \phi+u|\nabla u|^{2} \phi-\alpha|u|^{p-2} u \phi-\beta|u|^{q-2} u \phi\right] d x=0,
$$

for all $\phi \in C_{0}^{\infty}(\Omega)$, which is formally the variational formulation of the following functional

$$
I_{0}(u)=\frac{1}{2} \int_{\Omega}\left(1+u^{2}\right)|\nabla u|^{2} d x-\frac{\alpha}{p} \int_{\Omega}|u|^{p} d x-\frac{\beta}{q} \int_{\Omega}|u|^{q} d x .
$$

We may define the derivative of $I_{0}$ at $u$ in the direction of $\phi \in C_{0}^{\infty}(\Omega)$ as follows

$$
\left\langle I_{0}^{\prime}(u), \phi\right\rangle=\int_{\Omega}\left[\left(1+u^{2}\right) \nabla u \nabla \phi+u|\nabla u|^{2} \phi\right] d x
$$




$$
-\alpha \int_{\Omega}|u|^{p-2} u \phi d x-\beta \int_{\Omega}|u|^{q-2} u \phi d x .
$$

We call $u$ a critical point of $I_{0}$ if $u \in W_{0}^{1,2}(\Omega), \int_{\Omega} u^{2}|\nabla u|^{2} d x<\infty$ and $\left\langle I_{0}^{\prime}(u), \phi\right\rangle=$ 0 for all $\phi \in C_{0}^{\infty}(\Omega)$. That is, $u$ is a weak solution of $(P)$.

The main difficulty in our problems is that there is no suitable space on which the functional $I_{0}$ enjoys both smoothness and compactness, so the standard critical point theory can not be applied directly. To overcome this difficulty, we use a perturbation method developed recently in [22]. The main idea is, to find a family of $C^{1}$-functionals $I_{\mu}$ with compactness on a suitable work space, adding a perturbation term to the original functional $I_{0}$. So, we seek for a sequence $\left\{u_{\mu, n}\right\}$ of critical points of $I_{\mu}$ for $\mu>0$ small via the arguments in fountain theorem (see [4], [5], [6] and [26]) and establish suitable estimates for the critical points as $\mu \rightarrow 0$, and hence we may pass to the limit to get a sequence of solutions of the original problem. More precisely, we consider a family of perturbed functionals

$$
I_{\mu}(u)=\frac{\mu}{4} \int_{\Omega}|\nabla u|^{4} d x+I_{0}(u)
$$

where $\mu \in(0,1]$ is a parameter. Obviously, $I_{\mu}$ is a $C^{1}$-functional on $W_{0}^{1,4}(\Omega)$. For all $\phi \in W_{0}^{1,4}(\Omega)$,

$$
\left\langle I_{\mu}^{\prime}(u), \phi\right\rangle=\mu \int_{\Omega}|\nabla u|^{2} \nabla u \nabla \phi d x+\left\langle I_{0}^{\prime}(u), \phi\right\rangle .
$$

We have the following existence results for $(\mathrm{P})$.

Theorem 1.1. Assume $4<q<22^{*}$.

(a) For every $\beta>0, \alpha \in \mathbb{R}$, the problem $(\mathrm{P})$ has a sequence of weak solutions $\left\{u_{n}\right\}$ such that $I_{0}\left(u_{n}\right) \rightarrow \infty$ as $n \rightarrow \infty$.

(b) For every $\alpha>0, \beta \in \mathbb{R}$, the problem $(\mathrm{P})$ has a sequence of weak solutions $\left\{v_{n}\right\}$ with $I_{0}\left(v_{n}\right)<0$ such that $I_{0}\left(v_{n}\right) \rightarrow 0$ as $n \rightarrow \infty$.

TheOrem 1.2. Assume $q=22^{*}$ and $\beta>0$. Then there exists $\alpha^{*}>0$ such that, for every $0<\alpha<\alpha^{*}$, the problem (P) has a sequence of weak solutions $\left\{v_{n}\right\}$ with $I_{0}\left(v_{n}\right)<0$ such that $I_{0}\left(v_{n}\right) \rightarrow 0$ as $n \rightarrow \infty$.

REMARK 1.3. In fact, our results can be generalized to the more general case

$$
-\sum_{i, j=1}^{N} D_{j}\left(a_{i j}(x, u) D_{i} u\right)+\frac{1}{2} \sum_{i, j=1}^{N} D_{s} a_{i j}(x, u) D_{i} u D_{j} u=\alpha|u|^{p-2} u+\beta|u|^{q-2} u
$$

for $x \in \Omega$, where

$$
D_{i}=\frac{\partial}{\partial x_{i}} \quad \text { and } \quad D_{s} a_{i j}(x, s)=\frac{\partial}{\partial s} a_{i j}(x, s) .
$$

For $a_{i j}(x, u)=\left(1+u^{2}\right) \delta_{i j}$, the equation is reduced to $(\mathrm{P})$. 
Notations. We denote by $\|\cdot\|$ the norm of $W_{0}^{1,4}(\Omega)$, by $\|\cdot\|_{2}$ the norm of $W_{0}^{1,2}(\Omega)$ and by $|\cdot|_{s}$ the norm of $L^{s}(\Omega)(1<s<+\infty), C$ and $C_{i}$ stand for different positive constants.

\section{Proof of Theorem 1.1}

First, similar to [22], we have the following convergence results for (P).

Lemma 2.1. Let $\mu_{n} \rightarrow 0$ and $q \leq 22^{*}$. Suppose $\left\{u_{n}\right\} \subset W_{0}^{1,4}(\Omega)$ satisfies $I_{\mu_{n}}^{\prime}\left(u_{n}\right)=0$ and $I_{\mu_{n}}\left(u_{n}\right) \leq C$ for some $C \in \mathbb{R}$ independent of $n$. Then there is $u \in W_{0}^{1,4}(\Omega)$ such that, up to a subsequence, $u_{n} \rightarrow u$ in $W_{0}^{1,2}(\Omega), u_{n} \nabla u_{n} \rightarrow u \nabla u$ in $L^{2}(\Omega), \mu_{n} \int_{\Omega}\left|\nabla u_{n}\right|^{4} d x \rightarrow 0$ and $I_{\mu_{n}}\left(u_{n}\right) \rightarrow I_{0}(u)$ as $n \rightarrow \infty$, and $u$ is a critical point of $I_{0}$.

Proof. The proof is similar to [22]. We sketch it for completeness. By $I_{\mu_{n}}^{\prime}\left(u_{n}\right)=0$ and $I_{\mu_{n}}\left(u_{n}\right) \leq C$, we obtain

$$
\begin{aligned}
C \geq & I_{\mu_{n}}\left(u_{n}\right)-\frac{1}{q}\left\langle I_{\mu_{n}}^{\prime}\left(u_{n}\right), u_{n}\right\rangle \\
= & \left(\frac{1}{4}-\frac{1}{q}\right) \mu_{n} \int_{\Omega}\left|\nabla u_{n}\right|^{4} d x+\left(\frac{1}{2}-\frac{1}{q}\right) \int_{\Omega}\left|\nabla u_{n}\right|^{2} d x \\
& +\left(\frac{1}{2}-\frac{2}{q}\right) \int_{\Omega} u_{n}^{2}\left|\nabla u_{n}\right|^{2} d x+\alpha\left(\frac{1}{q}-\frac{1}{p}\right) \int_{\Omega}\left|u_{n}\right|^{p} d x .
\end{aligned}
$$

We claim that there exists $C_{0}>0$ such that

$$
\int_{\Omega}\left|u_{n}\right|^{p} d x \leq C_{0}
$$

If not, without loss of generality, we may assume $\left|u_{n}\right|_{p} \rightarrow \infty$ as $n \rightarrow \infty$. By (2.1), we have

$$
\begin{aligned}
C & \geq\left(\frac{1}{2}-\frac{2}{q}\right) \int_{\Omega} u_{n}^{2}\left|\nabla u_{n}\right|^{2} d x+\alpha\left(\frac{1}{q}-\frac{1}{p}\right)\left|u_{n}\right|_{p}^{p} \\
& =\left(\frac{1}{8}-\frac{1}{2 q}\right) \int_{\Omega}\left|\nabla u_{n}^{2}\right|^{2} d x+\alpha\left(\frac{1}{q}-\frac{1}{p}\right)\left|u_{n}\right|_{p}^{p} \\
& \geq\left(\frac{1}{8}-\frac{1}{2 q}\right) C_{1}\left|u_{n}\right|_{4}^{4}+\alpha\left(\frac{1}{q}-\frac{1}{p}\right)\left|u_{n}\right|_{p}^{p} \\
& \geq\left(\frac{1}{8}-\frac{1}{2 q}\right) C_{2}\left|u_{n}\right|_{p}^{4}-\alpha\left(\frac{1}{p}-\frac{1}{q}\right)\left|u_{n}\right|_{p}^{p},
\end{aligned}
$$

which is impossible since $p<2$ and $q>4$.

Using (2.1) again, we have

$$
\mu_{n} \int_{\Omega}\left|\nabla u_{n}\right|^{4} d x+\int_{\Omega}\left|\nabla u_{n}\right|^{2} d x+\int_{\Omega} u_{n}^{2}\left|\nabla u_{n}\right|^{2} d x \leq C_{3},
$$

where $C_{3}$ is independent of $n$. Then we have $u_{n} \rightarrow u$ in $W_{0}^{1,2}(\Omega), u_{n} \nabla u_{n} \rightarrow u \nabla u$ in $L^{2}(\Omega)$ and $u_{n}(x) \rightarrow u(x)$ for almost every $x \in \Omega$. Note that $u_{n}$ satisfies 


$$
\begin{aligned}
\mu_{n} \int_{\Omega}\left|\nabla u_{n}\right|^{2} \nabla u_{n} \nabla \phi d x & +\int_{\Omega}\left[\left(1+u_{n}^{2}\right) \nabla u_{n} \nabla \phi+u_{n}\left|\nabla u_{n}\right|^{2} \phi\right] d x \\
& -\alpha \int_{\Omega}\left|u_{n}\right|^{p-2} u_{n} \phi d x-\beta \int_{\Omega}\left|u_{n}\right|^{q-2} u_{n} \phi d x=0,
\end{aligned}
$$

for all $\phi \in W_{0}^{1,4}(\Omega)$. Since

$$
\left(\int_{\Omega}\left|u_{n}\right|^{4 N /(N-2)} d x\right)^{(N-2) / N} \leq C_{4} \int_{\Omega} u_{n}^{2}\left|\nabla u_{n}\right|^{2} d x \leq C_{5}
$$

by Moser's iteration we obtain

$$
\left|u_{n}\right|_{L^{\infty}(\Omega)} \leq C_{6},
$$

and hence $|u|_{L^{\infty}(\Omega)} \leq C_{6}$, where $C_{6}$ is independent of $n$. Now, similar to the arguments in [10] (see also [22]), one can show that $u$ is a critical point of $I_{0}$. In fact, we choose $\phi=\psi e^{-u_{n}}$ in (2.4), where $\psi \in C_{0}^{\infty}(\Omega)$ satisfies $\psi \geq 0$. It follows from (2.4) that

$$
\begin{aligned}
0= & \mu_{n} \int_{\Omega}\left|\nabla u_{n}\right|^{2} \nabla u_{n}\left(\nabla \psi e^{-u_{n}}-\psi \nabla u_{n} e^{-u_{n}}\right) d x \\
& +\int_{\Omega}\left(1+u_{n}^{2}\right) \nabla u_{n}\left(\nabla \psi e^{-u_{n}}-\psi \nabla u_{n} e^{-u_{n}}\right) d x \\
& +\int_{\Omega} u_{n}\left|\nabla u_{n}\right|^{2} \psi e^{-u_{n}} d x-\alpha \int_{\Omega}\left|u_{n}\right|^{p-2} u_{n} \psi e^{-u_{n}} d x \\
& -\beta \int_{\Omega}\left|u_{n}\right|^{q-2} u_{n} \psi e^{-u_{n}} d x \\
\leq & \mu_{n} \int_{\Omega}\left|\nabla u_{n}\right|^{2} \nabla u_{n} \nabla \psi e^{-u_{n}} d x+\int_{\Omega}\left(1+u_{n}^{2}\right) \nabla u_{n} \nabla \psi e^{-u_{n}} d x \\
& -\int_{\Omega}\left(1+u_{n}^{2}-u_{n}\right)\left|\nabla u_{n}\right|^{2} \psi e^{-u_{n}} d x \\
& -\alpha \int_{\Omega}\left|u_{n}\right|^{p-2} u_{n} \psi e^{-u_{n}} d x-\beta \int_{\Omega}\left|u_{n}\right|^{q-2} u_{n} \psi e^{-u_{n}} d x .
\end{aligned}
$$

By Fatou's lemma, the weak convergence of $u_{n}$ and (2.3) we have

$$
\begin{aligned}
0 \leq & \int_{\Omega}\left(1+u^{2}\right) \nabla u \nabla \psi e^{-u} d x-\int_{\Omega}\left(1+u^{2}-u\right)|\nabla u|^{2} \psi e^{-u} d x \\
& -\alpha \int_{\Omega}|u|^{p-2} u \psi e^{-u} d x-\beta \int_{\Omega}|u|^{q-2} u \psi e^{-u} d x \\
= & \int_{\Omega}\left(1+u^{2}\right) \nabla u \nabla\left(\psi e^{-u}\right) d x+\int_{\Omega} u|\nabla u|^{2} \psi e^{-u} d x \\
& -\alpha \int_{\Omega}|u|^{p-2} u \psi e^{-u} d x-\beta \int_{\Omega}|u|^{q-2} u \psi e^{-u} d x .
\end{aligned}
$$

Let $\chi \geq 0, \chi \in C_{0}^{\infty}(\Omega)$. We may choose a sequence of nonnegative functions $\psi_{n} \rightarrow \chi e^{u}$ in $W_{0}^{1,2}(\Omega), \psi_{n} \rightarrow \chi e^{u}$ for almost every $x \in \Omega$ and $\left\{\psi_{n}\right\}$ is uniformly bounded in $L^{\infty}(\Omega)$. Then, by approximations in (2.7), we have 


$$
\begin{aligned}
\int_{\Omega}\left(1+u^{2}\right) \nabla u \nabla \chi d x+\int_{\Omega} u|\nabla u|^{2} \chi d x & \\
& -\alpha \int_{\Omega}|u|^{p-2} u \chi d x-\beta \int_{\Omega}|u|^{q-2} u \chi d x \geq 0 .
\end{aligned}
$$

Similarly, we can obtain an opposite inequality. Thus, we have for all $\chi \in C_{0}^{\infty}(\Omega)$,

$$
\begin{aligned}
\int_{\Omega}\left(1+u^{2}\right) \nabla u \nabla \chi d x+\int_{\Omega} u|\nabla u|^{2} \chi d x & \\
& -\alpha \int_{\Omega}|u|^{p-2} u \chi d x-\beta \int_{\Omega}|u|^{q-2} u \chi d x=0 .
\end{aligned}
$$

That is, $u$ is a critical point of $I_{0}$ and a solution of $(\mathrm{P})$. Replacing $\chi$ with $u$ in (2.9) and doing approximations again we have

$$
\int_{\Omega}\left(1+u^{2}\right)|\nabla u|^{2} d x-\alpha \int_{\Omega}|u|^{p} d x-\beta \int_{\Omega}|u|^{q} d x=0 .
$$

Setting $\phi=u_{n}$ in (2.4), we have

$$
\mu_{n} \int_{\Omega}\left|\nabla u_{n}\right|^{4} d x+\int_{\Omega}\left(1+u_{n}^{2}\right)\left|\nabla u_{n}\right|^{2} d x-\alpha \int_{\Omega}\left|u_{n}\right|^{p} d x-\beta \int_{\Omega}\left|u_{n}\right|^{q} d x=0 .
$$

Using

$$
\int_{\Omega}\left|u_{n}\right|^{p} d x \rightarrow \int_{\Omega}|u|^{p} d x, \quad \int_{\Omega}\left|u_{n}\right|^{q} d x \rightarrow \int_{\Omega}|u|^{q} d x
$$

(2.10), (2.11) and the lower semi-continuity we obtain

$$
\begin{gathered}
\int_{\Omega}\left|\nabla u_{n}\right|^{2} d x \rightarrow \int_{\Omega}|\nabla u|^{2} d x, \quad \int_{\Omega} u_{n}^{2}\left|\nabla u_{n}\right|^{2} d x \rightarrow \int_{\Omega} u^{2}|\nabla u|^{2} d x, \\
\mu_{n} \int_{\Omega}\left|\nabla u_{n}\right|^{4} d x \rightarrow 0 .
\end{gathered}
$$

In particular, we have $u_{n} \rightarrow u$ in $W_{0}^{1,2}(\Omega), u_{n} \nabla u_{n} \rightarrow u \nabla u$ in $L^{2}(\Omega)$ and $I_{\mu_{n}}\left(u_{n}\right) \rightarrow I_{0}(u)$.

Let $\left\{e_{j}\right\}$ be a Schauder basis of $W_{0}^{1,4}(\Omega)$ (see [16] and [7]). Define $X_{j}:=\mathbb{R} e_{j}$. Note that for each $\mu \in(0,1], I_{\mu}$ is even. Now, some notations are in order. Set

$$
\begin{aligned}
Y_{k} & :=\bigoplus_{j=0}^{k} X_{j}, \quad Z_{k}:=\bigoplus_{j=k}^{\infty} X_{j}, \\
B_{k} & :=\left\{u \in Y_{k}: \int_{\Omega}\left(1+u^{2}\right)|\nabla u|^{2} d x \leq \rho_{k}^{2}\right\}, \\
N_{k} & :=\left\{u \in Z_{k}: \int_{\Omega}\left(1+u^{2}\right)|\nabla u|^{2} d x=r_{k}^{2}\right\},
\end{aligned}
$$

where $\rho_{k}>r_{k}>0$.

The following intersection property is similar to Lemma 3.4 in [26].

Lemma 2.2. If $\gamma \in C\left(B_{k}, W_{0}^{1,4}(\Omega)\right)$ is odd and $\left.\gamma\right|_{\partial B_{k}}=\mathrm{id}$, then

$$
\gamma\left(B_{k}\right) \cap N_{k} \neq \emptyset \text {. }
$$


Proof. Define

$$
U:=\left\{u \in B_{k}: \int_{\Omega}\left(1+\gamma^{2}(u)\right)|\nabla \gamma(u)|^{2} d x<r_{k}^{2}\right\} .
$$

Denote by $P_{k}$ the projector onto $Y_{k-1}$ such that $P_{k} Z_{k}=\{0\}$. By the BorsukUlam Theorem, there is $u_{0} \in B_{k}$ with

$$
\int_{\Omega}\left(1+\gamma^{2}\left(u_{0}\right)\right)\left|\nabla \gamma\left(u_{0}\right)\right|^{2} d x=r_{k}^{2}
$$

such that $P_{k} \gamma\left(u_{0}\right)=0$. Hence $u_{0} \in \gamma\left(B_{k}\right) \cap N_{k}$.

Proof of Theorem 1.1. (a) Assume $\alpha \in \mathbb{R}$ and $\beta>0$. The proof is divided into several steps.

Step 1. For each $k$, there is $r_{k}>0$ independent of $\mu \in(0,1]$ such that

$$
\inf _{u \in N_{k}} I_{\mu}(u) \rightarrow \infty, \quad \text { as } k \rightarrow \infty .
$$

In fact, define

$$
\theta_{k}:=\sup _{\substack{u \in Z_{k} \\ u \neq 0}} \frac{|u|_{q}^{2}}{\int_{\Omega}\left(1+u^{2}\right)|\nabla u|^{2} d x}
$$

It is clear that $0<\theta_{k+1} \leq \theta_{k}$, thus $\theta_{k} \rightarrow \theta \geq 0$ as $k \rightarrow \infty$. For each $k$, there exists $u_{k} \in Z_{k}$ such that

$$
\left(\int_{\Omega}\left(1+u_{k}^{2}\right)\left|\nabla u_{k}\right|^{2} d x\right)^{1 / 2}=1 \text { and }\left|u_{k}\right|_{q}>\frac{\theta_{k}}{2} .
$$

By the definition of $Z_{k}, u_{k} \rightarrow 0$ in $W_{0}^{1,4}(\Omega)$ (see p. 182-183 in [15]). The Sobolev imbedding theorem implies that $u_{k} \rightarrow 0$ in $L^{q}(\Omega)$. Therefore, $\theta=0$, i.e.

$$
\theta_{k} \rightarrow 0 \quad \text { as } k \rightarrow \infty \text {. }
$$

Observe that there exists $C_{1}>0$ such that $|u|^{p} \leq C_{1}\left(1+|u|^{q}\right)$, which yields that

$$
\begin{aligned}
I_{0}(u) & =\frac{1}{2} \int_{\Omega}\left(1+u^{2}\right)|\nabla u|^{2} d x-\frac{\alpha}{p}|u|_{p}^{p}-\frac{\beta}{q}|u|_{q}^{q} \\
& \geq \frac{1}{2} r_{k}^{2}-\frac{|\alpha| C_{1}|\Omega|}{p}-\left(\frac{\beta}{q}+\frac{|\alpha| C_{1}}{p}\right) \theta_{k}^{q}\left(\int_{\Omega}\left(1+u^{2}\right)|\nabla u|^{2} d x\right)^{q / 2} \\
& =\frac{1}{2} r_{k}^{2}-\frac{|\alpha| C_{1}|\Omega|}{p}-\left(\frac{\beta}{q}+\frac{|\alpha| C_{1}}{p}\right) \theta_{k}^{q} r_{k}^{q} \\
& =r_{k}^{2}\left[\frac{1}{2}-\left(\frac{\beta}{q}+\frac{|\alpha| C_{1}}{p}\right) \theta_{k}^{q} r_{k}^{q-2}\right]-\frac{|\alpha| C_{1}|\Omega|}{p}
\end{aligned}
$$

for $u \in N_{k}$. Choosing $r_{k}=\left[4\left(\beta / q+|\alpha| C_{1} / p\right)\right]^{-1 /(q-2)} \theta_{k}^{-q /(q-2)}$, we have $r_{k} \rightarrow \infty$ as $k \rightarrow \infty$. Thus

$$
\inf _{u \in N_{k}} I_{\mu}(u) \geq \inf _{u \in N_{k}} I_{0}(u) \geq \frac{1}{4} r_{k}^{2}-\frac{|\alpha| C_{1}|\Omega|}{p} \rightarrow \infty \quad \text { as } k \rightarrow \infty .
$$


Step 2. For each $k$, there is $\rho_{k}>r_{k}$ independent of $\mu \in(0,1]$ such that

$$
a_{k}:=\max _{\substack{u \in Y_{k} \\ \int_{\Omega}\left(1+u^{2}\right)|\nabla u|^{2}} x=\rho_{k}^{2}} I_{\mu}(u) \leq 0 .
$$

In fact, for $u \in Y_{k}$, we have

$$
\begin{aligned}
I_{\mu}(u)= & \frac{1}{4} \mu \int_{\Omega}|\nabla u|^{4} d x+\frac{1}{2} \int_{\Omega}\left(1+u^{2}\right)|\nabla u|^{2} d x \\
& -\frac{\alpha}{p} \int_{\Omega}|u|^{p} d x-\frac{\beta}{q} \int_{\Omega}|u|^{q} d x \\
\leq & \frac{1}{4} \mu \int_{\Omega}|\nabla u|^{4} d x+\frac{1}{2} \int_{\Omega}|\nabla u|^{2} d x+\frac{1}{2}\left(\int_{\Omega}|u|^{4} d x\right)^{1 / 2}\left(\int_{\Omega}|\nabla u|^{4} d x\right)^{1 / 2} \\
& +\frac{|\alpha|}{p} \int_{\Omega}|u|^{p} d x-\frac{\beta}{q} \int_{\Omega}|u|^{q} d x \\
\leq & \frac{1}{4} \mu\|u\|^{4}+C_{2}\|u\|^{2}+C_{3}\|u\|^{4}+C_{4}\|u\|^{p}-C_{5}\|u\|^{q} \\
\leq & \frac{1}{4}\|u\|^{4}+C_{2}\|u\|^{2}+C_{3}\|u\|^{4}+C_{4}\|u\|^{p}-C_{5}\|u\|^{q},
\end{aligned}
$$

since all norms are equivalent on the finite dimensional space $Y_{k}$, which implies that we can choose $\rho_{k}>r_{k}$ independent of $\mu \in(0,1]$ such that $b_{k} \leq 0$.

Step 3. We claim that $I_{\mu}$ satisfies the $(\mathrm{PS})_{c}$ condition for every $c>0$. In fact, let $\left\{u_{n}\right\} \subset W_{0}^{1,4}(\Omega)$ be such that $I_{\mu}\left(u_{n}\right) \rightarrow c, I_{\mu}^{\prime}\left(u_{n}\right) \rightarrow 0$ as $n \rightarrow \infty$. For $n$ large enough, we have

$$
\begin{aligned}
c+1+\left\|u_{n}\right\| \geq & I_{\mu}\left(u_{n}\right)-\frac{1}{q}\left\langle I_{\mu}^{\prime}\left(u_{n}\right), u_{n}\right\rangle \\
= & \left(\frac{1}{4}-\frac{1}{q}\right) \mu \int_{\Omega}\left|\nabla u_{n}\right|^{4} d x+\left(\frac{1}{2}-\frac{1}{q}\right) \int_{\Omega}\left|\nabla u_{n}\right|^{2} d x \\
& +\left(\frac{1}{2}-\frac{2}{q}\right) \int_{\Omega} u_{n}^{2}\left|\nabla u_{n}\right|^{2} d x+\left(\frac{\alpha}{q}-\frac{\alpha}{p}\right) \int_{\Omega}\left|u_{n}\right|^{p} d x \\
\geq & \left(\frac{1}{4}-\frac{1}{q}\right) \mu\left\|u_{n}\right\|^{4}-C_{6}\left\|u_{n}\right\|^{p} .
\end{aligned}
$$

Thus $\left\{u_{n}\right\}$ is bounded in $W_{0}^{1,4}(\Omega)$. Up to a subsequence, we may assume $u_{n} \rightarrow u$ in $W_{0}^{1,4}(\Omega)$ and $u_{n} \rightarrow u$ in $L^{s}(\Omega)$ for $1<s<22^{*}$. Choosing $\phi=u_{n}-u_{m}$ in (1.6) we have

$$
\begin{aligned}
o(1)\left\|u_{n}-u_{m}\right\|= & \left\langle I_{\mu}^{\prime}\left(u_{n}\right)-I_{\mu}^{\prime}\left(u_{m}\right), u_{n}-u_{m}\right\rangle \\
= & \mu \int_{\Omega}\left(\left|\nabla u_{n}\right|^{2} \nabla u_{n}-\left|\nabla u_{m}\right|^{2} \nabla u_{m}\right)\left(\nabla u_{n}-\nabla u_{m}\right) d x \\
& +\int_{\Omega}\left|\nabla u_{n}-\nabla u_{m}\right|^{2} d x \\
& +\int_{\Omega}\left(u_{n}^{2} \nabla u_{n}-u_{m}^{2} \nabla u_{m}\right)\left(\nabla u_{n}-u_{m}\right) d x
\end{aligned}
$$




$$
\begin{aligned}
& +\int_{\Omega}\left(u_{n}\left|\nabla u_{n}\right|^{2}-u_{m}\left|\nabla u_{m}\right|^{2}\right)\left(u_{n}-u_{m}\right) d x \\
& -\alpha \int_{\Omega}\left(\left|u_{n}\right|^{p-2} u_{n}-\left|u_{m}\right|^{p-2} u_{m}\right)\left(u_{n}-u_{m}\right) d x \\
& -\beta \int_{\Omega}\left(\left|u_{n}\right|^{q-2} u_{n}-\left|u_{m}\right|^{q-2} u_{m}\right)\left(u_{n}-u_{m}\right) d x .
\end{aligned}
$$

Now we estimate the above terms appeared on the right hand one by one.

$$
\begin{aligned}
& \int_{\Omega}\left(u_{n}^{2} \nabla u_{n}-u_{m}^{2} \nabla u_{m}\right)\left(\nabla u_{n}-u_{m}\right) d x \\
& =\int_{\Omega} u_{n}^{2}\left|\nabla u_{n}-\nabla u_{m}\right|^{2} d x+\int_{\Omega}\left(u_{n}^{2}-u_{m}^{2}\right) \nabla u_{m}\left(\nabla u_{n}-\nabla u_{m}\right) d x \\
& \geq-\left|u_{n}-u_{m}\right|_{4}\left(\left|u_{n}\right|_{4}+\left|u_{m}\right|_{4}\right)\left\|u_{m}\right\|\left(\left\|u_{n}\right\|+\left\|u_{m}\right\|\right) \rightarrow 0 .
\end{aligned}
$$

$$
\begin{aligned}
\left|\int_{\Omega}\left(u_{n}\left|\nabla u_{n}\right|^{2}-u_{m}\left|\nabla u_{m}\right|^{2}\right)\left(u_{n}-u_{m}\right) d x\right| \\
\leq\left(\left|u_{n}\right|_{4}\left\|u_{n}\right\|^{2}+\left|u_{m}\right|_{4}\left\|u_{m}\right\|^{2}\right)\left(\left|u_{n}-u_{m}\right|_{4}\right) \rightarrow 0 .
\end{aligned}
$$

$$
\begin{aligned}
& \left|\alpha \int_{\Omega}\left(\left|u_{n}\right|^{p-2} u_{n}-\left|u_{m}\right|^{p-2} u_{m}\right)\left(u_{n}-u_{m}\right) d x\right| \\
& \leq|\alpha|\left(\left|u_{n}\right|_{p}^{p-1}+\left|u_{m}\right|_{p}^{p-1}\right)\left|u_{n}-u_{m}\right|_{p} \rightarrow 0 \\
& \left|\beta \int_{\Omega}\left(\left|u_{n}\right|^{q-2} u_{n}-\left|u_{m}\right|^{q-2} u_{m}\right)\left(u_{n}-u_{m}\right) d x\right| \\
& \leq|\beta| \int_{\Omega}\left(\left|u_{n}\right|^{q-1}+\left|u_{m}\right|^{q-1}\right)\left|u_{n}-u_{m}\right| d x \\
& \leq|\beta|\left(\left|u_{n}\right|_{q}^{q-1}+\left|u_{m}\right|_{q}^{q-1}\right)\left|u_{n}-u_{m}\right|_{q} \rightarrow 0
\end{aligned}
$$

$$
\begin{aligned}
\mu \int_{\Omega}\left(\left|\nabla u_{n}\right|^{2} \nabla u_{n}-\left|\nabla u_{m}\right|^{2} \nabla u_{m}\right)\left(\nabla u_{n}-\nabla u_{m}\right) d x & \\
& \geq C_{7} \int_{\Omega}\left|\nabla u_{n}-\nabla u_{m}\right|^{4} d x
\end{aligned}
$$

for some $C_{7}>0$. Combining (2.16)-(2.20) together we obtain

$$
C_{7} \int_{\Omega}\left|\nabla u_{n}-\nabla u_{m}\right|^{4} d x \leq o(1)\left\|u_{n}-u_{m}\right\|+o(1),
$$

which impliese that $\left\{u_{n}\right\}$ is a Cauchy sequence in $W_{0}^{1,4}(\Omega)$, and hence there is $u \in W_{0}^{1,4}(\Omega)$ such that $u_{n} \rightarrow u$ in $W_{0}^{1,4}(\Omega)$.

Step 4. Define for $k \geq 2, c_{k}(\mu):=\inf _{\gamma \in \Gamma_{k}} \max _{u \in B_{k}} I_{\mu}(\gamma(u))$, where

$$
\Gamma_{k}:=\left\{\gamma \in C\left(B_{k}, X\right): \gamma \text { is odd and }\left.\gamma\right|_{\partial B_{k}}=\mathrm{id}\right\} .
$$


Obviously, for each $k$, there holds $c_{k}(\mu) \leq c_{k}(1) \leq \max _{u \in B_{k}} I_{1}(u)<\infty$, since $I_{\mu}$ is increasing in $\mu$. On the other hand, by Lemma 2.2, $\gamma\left(B_{k}\right) \cap N_{k} \neq \emptyset$ for $\gamma \in \Gamma_{k}$. Therefore, $c_{k}(\mu) \geq \inf _{u \in N_{k}} I_{\mu}(u) \geq \inf _{u \in N_{k}} I_{0}(u)$. It follows from (2.13) that $c_{k}(\mu) \rightarrow \infty$ as $k \rightarrow \infty$.

Step 5. For each $k$, there is a sequence $u_{n, k}$ such that $I_{\mu}\left(u_{n, k}\right) \rightarrow c_{k}(\mu)$ and $I_{\mu}^{\prime}\left(u_{n, k}\right) \rightarrow 0$ as $n \rightarrow \infty$. Otherwise, for every $\varepsilon \in\left(0,\left(c_{k}-a_{k}\right) / 2\right)$ and $\gamma \in \Gamma_{k}$ with $\max _{u \in B_{k}} I_{\mu}(\gamma(u)) \leq c_{k}(\mu)+\varepsilon$, where $a_{k}$ is given in (2.14), it follows from the equivariant deformation lemma (see Lemma 3.1 in [26]) that there exists $\eta \in C\left([0,1] \times W_{0}^{1,4}(\Omega), W_{0}^{1,4}(\Omega)\right)$ such that $\eta\left(1, I_{\mu}^{c_{k}(\mu)+\varepsilon}\right) \subset I_{\mu}^{c_{k}(\mu)-\varepsilon}$ and $\varphi(u):=\eta(1, \gamma(u)) \in \Gamma_{k}$. Thus $c_{k}(\mu) \leq \max _{u \in B_{k}} I_{\mu}(\gamma(u)) \leq c_{k}(\mu)-\varepsilon$, which is absurd.

By Step 3, $c_{k}(\mu)$ is a critical value of $I_{\mu}$. Thus $I_{\mu}$ has an unbounded sequence of critical values and hence $I_{\mu}$ has a sequence of critical points $\left\{u_{\mu, k}\right\}$. By Lemma 2.1, passing to the limit for $\mu \rightarrow 0, I_{0}$ has a sequence of critical points $\left\{u_{k}\right\}$ such that $I_{0}\left(u_{k}\right) \rightarrow \infty$ as $k \rightarrow \infty$. This completes the proof of (a).

(b) Assume $\alpha>0$ and $\beta \in \mathbb{R}$. The proof is also divided into several steps.

Step 1. We claim there exists $k_{0}$ such that, for each $k \geq k_{0}$, there is $\rho_{k}>0$ independent of $\mu \in(0,1]$ such that

$$
\inf _{\substack{u \in Z_{k} \\ \int_{\Omega}\left(1+u^{2}\right)|\nabla u|^{2} d x=\rho_{k}^{2}}} I_{\mu}(u) \geq 0 .
$$

In fact, define

$$
\vartheta_{k}:=\sup _{\substack{u \in Z_{k} \\ u \neq 0}} \frac{|u|_{p}^{2}}{\int_{\Omega}\left(1+u^{2}\right)|\nabla u|^{2} d x} .
$$

Similar to (2.12), $\vartheta_{k} \rightarrow 0$ as $k \rightarrow \infty$. Since

$$
|u|_{q} \leq C_{0}\left(\int_{\Omega}|\nabla u|^{2} d x\right)^{1 / 2} \leq C_{0}\left(\int_{\Omega}\left(1+u^{2}\right)|\nabla u|^{2} d x\right)^{1 / 2}
$$

and $q>4$, there exists $R>0$ such that

$$
\int_{\Omega}\left(1+u^{2}\right)|\nabla u|^{2} d x \leq R \Rightarrow \frac{\beta}{q}|u|_{q}^{q} \leq \frac{1}{4} \int_{\Omega}\left(1+u^{2}\right)|\nabla u|^{2} d x
$$

which yields

$$
\begin{aligned}
I_{0}(u) & =\frac{1}{2} \int_{\Omega}\left(1+u^{2}\right)|\nabla u|^{2} d x-\frac{\alpha}{p}|u|_{p}^{p}-\beta / q|u|_{q}^{q} \\
& \geq \frac{1}{4} \int_{\Omega}\left(1+u^{2}\right)|\nabla u|^{2} d x-\alpha / p|u|_{p}^{p} \\
& \geq \frac{1}{4} \int_{\Omega}\left(1+u^{2}\right)|\nabla u|^{2} d x-\frac{\alpha}{p} \vartheta_{k}^{p}\left(\int_{\Omega}\left(1+u^{2}\right)|\nabla u|^{2} d x\right)^{p / 2},
\end{aligned}
$$

for $u \in W_{0}^{1,4}(\Omega)$ with $\int_{\Omega}\left(1+u^{2}\right)|\nabla u|^{2} d x \leq R$. 
Choosing $\rho_{k}=(8 \alpha / p)^{1 /(2-p)} \vartheta_{k}^{p /(2-p)}$ we have $\rho_{k} \rightarrow 0$ as $k \rightarrow \infty$. Then there exists $k_{0}$ such that $\rho_{k} \leq R$ when $k \geq k_{0}$. Thus, for $k \geq k_{0}$ and $u \in Z_{k}$ with $\int_{\Omega}\left(1+u^{2}\right)|\nabla u|^{2} d x=\rho_{k}^{2}$, it follows from (2.22) that

$$
I_{\mu}(u) \geq I_{0}(u) \geq \rho_{k}^{2}\left[\frac{1}{4}-\frac{\alpha}{p} \vartheta_{k}^{p} \rho_{k}^{p-2}\right] \geq 0,
$$

which implies that the claim is true.

Step 2. For each $k \geq k_{0}$, there is $0<r_{k}<\rho_{k}$ independent of $\mu \in(0,1]$ such that

$$
\bar{b}_{k}:=\max _{\substack{u \in Y_{k} \\ \int_{\Omega}\left(1+u^{2}\right)|\nabla u|^{2}}} I_{\mu}(u)<0 .
$$

In fact, for $u \in Y_{k}$, we have

$$
\begin{aligned}
I_{\mu}(u) & =\frac{\mu}{4} \int_{\Omega}|\nabla u|^{4} d x+\frac{1}{2} \int_{\Omega}\left(1+u^{2}\right)|\nabla u|^{2} d x-\frac{\alpha}{p} \int_{\Omega}|u|^{p} d x-\frac{\beta}{q} \int_{\Omega}|u|^{q} d x \\
& \leq \frac{\mu}{4}|\nabla u|_{4}^{4}+\frac{1}{2}|\nabla u|_{2}^{2}+\frac{1}{2}|u|_{4}^{2}|\nabla u|_{4}^{2}-\frac{\alpha}{p}|u|_{p}^{p}+\frac{|\beta|}{q}|u|_{q}^{q} \\
& \leq \frac{1}{4}\|u\|^{4}+C_{1}\|u\|^{2}+C_{2}\|u\|^{4}-C_{3}\|u\|^{p}+C_{4}\|u\|^{q},
\end{aligned}
$$

since all norms are equivalent on the finite dimensional space $Y_{k}$, which implies that we can choose $0<r_{k}<\rho_{k}$ independent of $\mu \in(0,1]$ such that $\bar{b}_{k}<0$.

Step 3. We obtain from (2.22), for $k \geq k_{0}$ and $u \in B_{k}$

$$
I_{\mu}(u) \geq I_{0}(u) \geq-\frac{\alpha}{p} \vartheta_{k}^{p}\left(\int_{\Omega}\left(1+u^{2}\right)|\nabla u|^{2} d x\right)^{p / 2} \geq-\frac{\alpha}{p} \vartheta_{k}^{p} \rho_{k}^{p} .
$$

Then $\bar{a}_{k}:=\inf _{u \in B_{k}} I_{\mu}(u) \rightarrow 0$ as $k \rightarrow \infty$ since $\vartheta_{k} \rightarrow 0$ and $\rho_{k} \rightarrow 0$ as $k \rightarrow \infty$.

Step 4. Now we prove that $I_{\mu}$ satisfies the $(\mathrm{PS})_{c}^{*}$ condition for each $c<0$ with respect to $\left\{Y_{k}\right\}$. Consider a sequence $\left\{u_{n_{k}}\right\} \subset W_{0}^{1,4}(\Omega)$ such that $n_{k} \rightarrow \infty$, $u_{n_{k}} \in Y_{n_{k}}, I_{\mu}\left(u_{n_{k}}\right) \rightarrow c$ and $\left.I_{\mu}\right|_{Y_{n_{k}}} ^{\prime}\left(u_{n_{k}}\right) \rightarrow 0$. For $k$ large enough, we have

$$
\begin{aligned}
c+1+\left\|u_{n_{k}}\right\| \geq & I_{\mu}\left(u_{n_{k}}\right)-\frac{1}{q}\left\langle I_{\mu}^{\prime}\left(u_{n_{k}}\right), u_{n_{k}}\right\rangle \\
= & \left(\frac{1}{4}-\frac{1}{q}\right) \mu \int_{\Omega}\left|\nabla u_{n_{k}}\right|^{4} d x+\left(\frac{1}{2}-\frac{1}{q}\right) \int_{\Omega}\left|\nabla u_{n_{k}}\right|^{2} d x \\
& +\left(\frac{1}{2}-\frac{2}{q}\right) \int_{\Omega} u_{n_{k}}^{2}\left|\nabla u_{n_{k}}\right|^{2} d x+\alpha\left(\frac{1}{q}-\frac{1}{p}\right) \int_{\Omega}\left|u_{n_{k}}\right|^{p} d x \\
\geq & \left(\frac{1}{4}-\frac{1}{q}\right) \mu\left\|u_{n_{k}}\right\|^{4}+\alpha\left(\frac{1}{q}-\frac{1}{p}\right) C_{7}\left\|u_{n_{k}}\right\|^{p} .
\end{aligned}
$$

Thus $\left\{u_{n_{k}}\right\}$ is bounded in $W_{0}^{1,4}(\Omega)$. Similar to the proof of part (a), one can show that $\left\{u_{n_{k}}\right\}$ has a convergent subsequence in $W_{0}^{1,4}(\Omega)$. 
Step 5. We fix $n \geq k \geq k_{0}$ and define

$$
\begin{aligned}
Z_{k}^{n} & :=\bigoplus_{j=k}^{n} X_{j}, \\
B_{k}^{n} & :=\left\{u \in Z_{k}^{n}: \int_{\Omega}\left(1+u^{2}\right)|\nabla u|^{2} d x \leq \rho_{k}^{2}\right\}, \\
\Gamma_{k}^{n} & :=\left\{\gamma \in C\left(B_{k}^{n}, Y_{n}\right): \gamma \text { is odd and }\left.\gamma\right|_{\partial B_{k}^{n}}=\mathrm{id}\right\}, \\
\bar{c}_{k}^{n}(\mu) & :=\sup _{\gamma \in \Gamma_{k}^{n}} \min _{u \in B_{k}^{n}} I_{\mu}(\gamma(u)) .
\end{aligned}
$$

Then $\bar{c}_{k}^{n}(\mu) \in\left[\bar{a}_{k}, \bar{b}_{k}\right]$. Now, repeating the arguments in (a) to the functional $-I_{\mu}$ defined on the space $Y_{n}$, there exists $u_{n} \in Y_{n}$ such that

$$
\bar{c}_{k}^{n}(\mu)-\frac{2}{n} \leq I_{\mu}\left(u_{n}\right) \leq \bar{c}_{k}^{n}(\mu)+\frac{2}{n}, \quad\left\|\left.I_{\mu}\right|_{X_{n}} ^{\prime}\left(u_{n}\right)\right\| \leq \frac{8}{n} .
$$

Since $I_{\mu}$ satisfies the $(\mathrm{PS})_{c}^{*}$ condition, we see that $\left\{\bar{c}_{k}^{n}(\mu)\right\}$ converges along a subsequence to a critical value $\bar{c}_{k}(\mu) \in\left[\bar{a}_{k}, \bar{b}_{k}\right]$ of $I_{\mu}$ as $n \rightarrow \infty$. Moreover, by Step $3, \bar{c}_{k}(\mu) \rightarrow 0_{-}$as $k \rightarrow \infty$. Using Lemma 2.1 and passing to the limit for $\mu \rightarrow 0$ we have that $I_{0}$ has a sequence of negative critical values going to 0 . This completes the proof of (b).

\section{Proof of Theorem 1.2}

This section is devoted to proving Theorem 1.2. The following lemma plays a key role in the proof.

Lemma 3.1. There is $\lambda>0$ such that, for any $\alpha>0$ and

$$
c<\frac{S^{N / 2}}{2 N 2^{N / 2} \beta^{(N-2) / 2}}-\lambda \alpha^{22^{*} /\left(22^{*}-p\right)},
$$

the functional $I_{\mu}$ satisfies the $(\mathrm{PS})_{c}^{*}$ condition.

Proof. Consider a sequence $\left\{u_{n_{k}}\right\} \subset W_{0}^{1,4}(\Omega)$ such that $n_{k} \rightarrow \infty, u_{n_{k}} \in$ $Y_{n_{k}}, I_{\mu}\left(u_{n_{k}}\right) \rightarrow c,\left.I_{\mu}\right|_{Y_{n_{k}}} ^{\prime}\left(u_{n_{k}}\right) \rightarrow 0$. As in the proof of Theorem 1.1, $\left\{u_{n_{k}}\right\}$ is bounded in $W_{0}^{1,4}(\Omega)$. Going if necessary to a subsequence, we can assume that $u_{n_{k}} \rightarrow u$ in $W_{0}^{1,4}(\Omega), u_{n_{k}} \rightarrow u$ in $L^{s}(\Omega)$ for $1<s<22^{*}$ and $u_{n_{k}} \rightarrow u$ almost everywhere on $\Omega$. Since $\left\{u_{n_{k}}\right\}$ is bounded in $L^{22^{*}}(\Omega),\left\{\left|u_{n_{k}}\right|^{22^{*}-2} u_{n_{k}}\right\}$ is bound in $L^{4 N /(3 N+2)}(\Omega)$ and so

$$
\left|u_{n_{k}}\right|^{22^{*}-2} u_{n_{k}} \rightarrow|u|^{22^{*}-2} u \quad \text { in } L^{4 N /(3 N+2)}(\Omega) .
$$

Then a standard argument shows that $u$ is a critical point of $I_{\mu}$ (see [26]).

We write $v_{n_{k}}:=u_{n_{k}}-u$. The Brezis-Lieb lemma (see [8]) leads to

$$
\begin{aligned}
& \left|\nabla u_{n_{k}}^{2}\right|_{2}^{2}=\left|\nabla u^{2}\right|_{2}^{2}+\left|\nabla v_{n_{k}}^{2}\right|_{2}^{2}+o(1), \\
& \left|u_{n_{k}}\right|_{22^{*}}^{22^{*}}=|u|_{22^{*}}^{22^{*}}+\left|v_{n_{k}}\right|_{22^{*}}^{22^{*}}+o(1) .
\end{aligned}
$$


Since $\left\langle I_{\mu}^{\prime}\left(u_{n_{k}}\right), u_{n_{k}}\right\rangle \rightarrow 0$, so we have

$$
\begin{aligned}
& \mu\left|\nabla v_{n_{k}}\right|_{4}^{4}+\left|\nabla v_{n_{k}}\right|_{2}^{2}+\frac{1}{2}\left|\nabla v_{n_{k}}^{2}\right|_{2}^{2}-\beta\left|v_{n_{k}}\right|_{22^{*}}^{22^{*}} \\
& \rightarrow-\mu \int_{\Omega}|\nabla u|^{4} d x-2 \int_{\Omega} u^{2}|\nabla u|^{2} d x-\int_{\Omega}|\nabla u|^{2} d x+\alpha \int_{\Omega}|u|^{p} d x+\beta \int_{\Omega}|u|^{22^{*}} d x \\
& =-\left\langle I_{\mu}^{\prime}(u), u\right\rangle=0 .
\end{aligned}
$$

Therefore, we may assume that

$$
\mu\left|\nabla v_{n_{k}}\right|_{4}^{4}+\left|\nabla v_{n_{k}}\right|_{2}^{2}+\frac{1}{2}\left|\nabla v_{n_{k}}^{2}\right|_{2}^{2} \rightarrow b, \quad \beta\left|v_{n_{k}}\right|_{22^{*}}^{22^{*}} \rightarrow b
$$

By the Soblev inequality, we have

$$
b \geq \frac{1}{2}\left|\nabla v_{n_{k}}^{2}\right|_{2}^{2} \geq \frac{1}{2} S\left|v_{n_{k}}^{2}\right|_{2^{*}}^{2}=\frac{1}{2} S\left|v_{n_{k}}\right|_{22^{*}}^{4},
$$

and so $b \geq S(b / \beta)^{2 / 2^{*}} / 2$. Then $b=0$ or $b \geq S^{N / 2} /\left(2^{N / 2} \beta^{(N-2) / 2}\right)$.

Assume $b \geq S^{N / 2} /\left(2^{N / 2} \beta^{(N-2) / 2}\right)$. We have

$$
\begin{aligned}
c+o(1) & =I_{\mu}\left(u_{n_{k}}\right)-\frac{1}{4}\left\langle I_{\mu}^{\prime}\left(u_{n_{k}}\right), u_{n_{k}}\right\rangle \\
& =\frac{1}{4}\left|\nabla u_{n_{k}}\right|_{2}^{2}+\alpha\left(\frac{1}{4}-\frac{1}{p}\right)\left|u_{n_{k}}\right|_{p}^{p}+\beta\left(\frac{1}{4}-\frac{1}{22^{*}}\right)\left|u_{n_{k}}\right|_{22^{*}}^{22^{*}} \\
& \geq \alpha\left(\frac{1}{4}-\frac{1}{p}\right)\left|u_{n_{k}}\right|_{p}^{p}+\frac{\beta}{2 N}\left|u_{n_{k}}\right|_{22^{*}}^{22^{*}} \\
& =\alpha\left(\frac{1}{4}-\frac{1}{p}\right)|u|_{p}^{p}+\frac{\beta}{2 N}\left(\frac{b}{\beta}+|u|_{22^{*}}^{22^{*}}\right)+o(1) \\
& \geq \frac{S^{N / 2}}{2 N 2^{N / 2} \beta^{(N-2) / 2}}+\frac{\beta}{2 N}|u|_{22^{*}}^{22^{*}}-C_{1} \alpha|u|_{22^{*}}^{p}
\end{aligned}
$$

for some $C_{1}>0$. A direct computation shows that

$$
\min _{t>0}\left(\frac{\beta}{2 N} t^{22^{*}}-C_{1} \alpha t^{p}\right)=-\left(1-\frac{p}{22^{*}}\right) C_{1}^{22^{*} /\left(22^{*}-p\right)}(p N)^{p /\left(22^{*}-p\right)} \alpha^{22^{*} /\left(22^{*}-p\right)}
$$

Setting $\lambda:=\left(1-p / 22^{*}\right) C_{1}^{22^{*} /\left(22^{*}-p\right)}(p l N)^{p /\left(22^{*}-p\right)}>0$ we have

$$
c \geq \frac{S^{N / 2}}{2 N 2^{N / 2} \beta^{(N-2) / 2}}-\lambda \alpha^{22^{*} /\left(22^{*}-p\right)},
$$

which contradicts (3.1). So $b=0$, and therefore $u_{n_{k}} \rightarrow u$ in $W_{0}^{1,4}(\Omega)$.

Proof of Theorem 1.2. By Lemma 3.1, there exists $\alpha^{*}>0$ such that for every $0<\alpha<\alpha^{*}$ and $c<0$, the functional $I_{\mu}(u)$ satisfies the $(\mathrm{PS})_{c}^{*}$ condition. Now, repeating the proof of the part (b) of Theorem 1.1 we can obtain the conclusion.

Acknowledgments. F. Zhao thanks the College of William and Mary for the invitation and the hospitality during his visit. 


\section{REFERENCES}

[1] C.O. Alves, G.M. Figueiredo and U.B. Severo, Multiplicity of positive solutions for a class of quasilinear problems, Adv. Differential Equations 14 (2009), 911-942.

[2] A. Ambrosetti, H. Brezis and G. Cerami, Combined effects of concave and convex nonlinearities in some elliptic problems, J. Funct. Anal. 122 (1994), 519-543.

[3] A. Ambrosetti And Z.-Q. WAng, Positive solutions to a class of quasilinear elliptic equations on $\mathbb{R}$, Discrete Contin. Dyn. Syst. 9 (2003), 55-66.

[4] T. BARTSCH, Infinitely many solutions of a symmetric Dirichlet problem, Nonlinear Anal. 20 (1993), 1205-1216.

[5] T. Bartsch And M. Willem, Periodic solutions of nonautonomous Hamiltonian systems with symmetries, J. Reine Angew. Math. 451 (1994), 149-159.

[6] - On an elliptic equation with concave and convex nonlinearities, Proc. Amer. Math. Soc. 123 (1995), 3555-3561.

[7] H. Bellout, On a special Schauder basis for the Sobolev spaces $W_{0}^{1, p}(\Omega)$, Illinois J. Math. 39 (1995), 187-195.

[8] H. Brezis And E. Lieb, A relation between pointwise convergence of functions and convergence of functionals, Proc. Amer. Math. Soc. 88 (1983), 486-490.

[9] H. Brezis and L. Nirenberg, Positive solutions of nonlinear elliptic equations involving critical sobolev exponents, Comm. Pure Appl. Math. 36 (1983), 437-477.

[10] A. Canino and M. Degiovanni, Nonsmooth critical point theory and quaslinear Schrödinger elliptic equations, Topol. Methods in Differential Equations and Equations and Inclusions (Montreal, PQ, 1994), 472 (1995), 1-50.

[11] M. Colin And L. Jeanjean, Solutions for quasilinear Schrödinger equations: a dual approach, Nonlinear Anal. 56 (2002), 392-344.

[12] J.M. Do Ó, O. MiYagaki ANd S. SoARES, Soliton solutions for quasilinear Schrödinger equations with critical growth, J. Differential Equations 248 (2010), 722-744.

[13] J.M. Do Ó AND U. SEvero, Quasilinear Schrödinger equations involving concave and convex nonlinearities, Commun. Pure Appl. Anal. 8 (2009), 621-644.

[14] _ Solitary wave for a class of quasilinear quasilinear Schrödinger equations in dimension two, Calc. Var. Partial Differential Equations 69 (2010), 397-408.

[15] M. Fabian, P. Habala, P. Hájek, V. Montesinos and V. Zizler, Banach Space Theory. The Basis for Linear and Nonlinear Analysis. CMS Books in Mathematics/Ouvrages de Mathématiques de la SMC, Springer, New York, 2011.

[16] S. Fučí́, O. John AND J. NeČAs, On the existence of Schauder bases in Sobolev spaces, Comment. Math. Univ. Carolinae 13 (1972), 163-175.

[17] A. J. García And A.I. Peral, Multiplicity of solutions for elliptic problems with critical exponent or with a nonsymmetric term, Trans. Amer. Math. Soc. 323 (1991), 877-895.

[18] H.F. Lins And E.A.B. Silva, Quasilinear asymptotically periodic elliptic equations with critical growth, Nonlinear Anal. 71 (2009), 2890-2905.

[19] J. Liu And Z.-Q. Wang, Solition solutions for quasilinear Schrödinger equations, Proc. Amer. Math. Soc. 131 (2003), 329-344.

[20] J. Liu, Y. WANG AND Z.-Q. WANG, Solutions for quasilinear Schrödinger equations via the Nehari method, Comm. Partial Differential Equations 29 (2004), 879-901.

[21] _ Solition solutions for quasilinear Schrödinger equations II, J. Differential Equations 187 (2003), 473-493.

[22] X. Liu, J. LiU AND Z.-Q. WANG, Quasilinear elliptic equations via perturbation method, Proc. Amer. Math. Soc. 141 (2013), 253-263. 
[23] A. MoAmeni, Existence of solition solutions for a quasilinear Schrödinger equations involing critical growth in $\mathbb{R}^{N}$, J. Differential Equations 229 (2006), 570-587.

[24] M. Poppenburg, K.Schmitt And Z.-Q. WAng, On the existence of solutions to quasilinear Schrödinger equations, Calc. Var. Partial Differential Equations 14 (2002), 392-344.

[25] E.A.B. Silva AND G.F. VIEIRA, Quasilinear asymptotically periodic Schrödinger equations with critical growth, Nonlinear Anal. 72 (2010), 2935-2949.

[26] M. Willem, Minimax Theorem, Progress in Nonlinear Differential Equations and their Applications, Vol. 24, Birkhäuser Boston, Inc., Boston, MA, 1996.

Manuscript received January 1, 2014

Leran Xia AND Fukun ZHaO

Department of Mathematics

Yunnan Normal University

Kunming 650500 Yunnan, P.R.CHINA

E-mail address: fukunzhao@163.com

MinBo YANG

Department of Mathematics

Zhejiang Normal University

Jinhua 321004 Zhejiang, P.R.CHINA

TMNA : VOLUME $44-2014-\mathrm{N}^{\mathrm{O}} 2$ 